\title{
OPADIA Study: Is a Patient Questionnaire Useful for Enhancing Physician-Patient Shared Decision Making on Physical Activity Micro-objectives in Diabetes?
}

\author{
Silla M. Consoli · Martine Duclos • André Grimaldi • Alfred Penfornis • \\ Sébastien Bineau · Bénédicte Sabin · Mathilde Pouriel • \\ Eva Freund · Sylvie Dejager
}

Received: February 14, 2020 / Published online: April 15, 2020

(C) The Author(s) 2020

\section{ABSTRACT}

Introduction: Regular physical activity (PA) is recommended by all type 2 diabetes mellitus (T2DM) management guidelines. The OPADIA study aimed to determine whether using a specific patient questionnaire (Optima-PA@) could help T2DM patients increase their PA by leading to better physician-patient communication and improved levels of shared decision making concerning Specific, Measurable, Acceptable, Realistic, Timely (SMART)-PA micro-objectives.

Digital Features To view digital features for this article go to https://doi.org/10.6084/m9.figshare.12063366.

Electronic supplementary material The online version of this article (https://doi.org/10.1007/s12325020-01336-8) contains supplementary material, which is available to authorized users.

S. M. Consoli

Paris Descartes University of Medicine, Sorbonne

Paris Cité University, Paris, France

\section{Duclos}

Department of Sport Medicine and Functional Explorations, University-Hospital (CHU), G. Montpied Hospital, INRA, UMR 1019, UNH, CRNH Auvergne, Clermont University, University of Auvergne, Clermont-Ferrand, France

A. Grimaldi

Service de diabétologie, Institut E3M, Hôpital Pitié-

Salpêtrière, Paris, France
Methods: Physicians participating in this multicentre, prospective, randomised, real-life study were allocated to a standard group (T2DM patients managed according to usual clinical practice, $n=24$ ) or the OPTIMA-PA group (additional use of the questionnaire, $n=30$ ). The main outcome was the percentage of inclusion visits ending with the setting up of at least one SMART-PA micro-objective. Other outcomes were the impact of the OPTIMA-PA questionnaire on patient perceptions of shared decision making (ENTRED questionnaire) and the impact of the OPTIMA-PA questionnaire and establishing SMART-PA micro-objectives as well as patient-perceived physician empathy (ENTRED questionnaire) and GP aptitude for patient-centredness (SEPCQ scores) on patient

\section{A. Penfornis}

Service d'endocrinologie, diabétologie et maladies métaboliques, Centre Hospitalier Sud-Francilien de Corbeil-Essonnes, Université Paris-Saclay, Orsay, France

S. Bineau $\cdot$ S. Dejager $(\bowtie)$

MSD France, Immeuble Carré Michelet, 10/12 Cours

Michelet, 92800 Puteaux, France

e-mail: sylvie.dejager@msd.com

B. Sabin · M. Pouriel

Kappa Santé, 4 rue de Cléry, 75002 Paris, France

E. Freund

Clintec International SARL, Tour D2, 17 bis place des Reflets, 92099 La Défense, France 
PA levels over a 3-month period (IPAQ-SF scores).

Results: One hundred twenty-two patients were included in the standard group and 134 in the OPTIMA-PA group. Unexpectedly, more inclusion visits ended with SMART-PA microobjectives being set up in the standard group $(p<0.001): 81.1 \%(n=99 / 122)$ versus $59.7 \%$ $(n=80 / 134)$. However, fewer patients in the OPTIMA-PA group felt that GPs made decisions alone $(32 \%$ versus $60 \% ; p<0.0001)$. Positive correlations were also observed between GP patient-centredness and patient-perceived GP empathy or increased patient PA over the study period.

Conclusion: Although the OPTIMA-PA questionnaire did not directly promote setting up of SMART-PA micro-objectives in T2DM patients, the OPADIA study demonstrated that this tool was effective at improving patient-physician relationships by increasing patient involvement in therapeutic decision making. Our study also highlighted the importance of GP aptitude for patient-centredness for improving PA in T2DM patients.

Keywords: Diabetes mellitus; Patient centredness; Patient questionnaire; Physical activity; Physician empathy; Shared decision making; Type 2

\section{Key Summary Points}

Why carry out this study?

Regular physical activity has demonstrated beneficial effects on glycaemic control, quality of life and T2DM-related comorbidities and is recommended by all guidelines.

The use of a specific patient questionnaire to improve physician-patient communication on physical activity in diabetes (Optima-PA@ questionnaire) might improve levels of shared decision making concerning attainable microobjectives for physical activity and thus help patients increase their physical activity.

\section{What was learned from the study?}

Use of the Optima-PA questionnaire did not increase the rate of inclusion visits ending with Specific, Measurable, Acceptable, Realistic, Timely (SMART)-PA micro-objectives being set up, but had a positive impact on patient perceptions of shared decision making.

The aptitude of the general practitioner for developing a patient-centred relationship was an important determinant for improving patient levels of physical activity.

\section{INTRODUCTION}

Adherence of patients with type 2 diabetes mellitus (T2DM), not only to their treatment but also to healthy lifestyle recommendations, is a key goal of T2DM management $[1,2]$. In particular, regular physical activity (PA) has been demonstrated to have beneficial effects on glycaemic control [improvement of glycated haemoglobin (HbA1c) levels] and T2DM-related cardiovascular risk factors (improvements in blood pressure values and lipid profiles and a decrease in insulin resistance) [3]. Regular PA is now recommended by all guidelines [2-4]. Levels of patient understanding of their diabetes, and involvement in defining their therapeutic objectives with their physician, correlate directly with levels of adherence to treatment and improvements in pathology (i.e., a decrease in the occurrence of complications) [5].

The OPTIMA $\odot$ (MSD, Courbevoie, France) questionnaire was developed in collaboration with T2DM patients, general practitioners (GPs) and diabetologists to enhance physician-patient communication on diabetes [6]. This questionnaire helps physicians gather information on the lifestyles of their patients and helps the patients express their preferences and their difficulties in following the prescriptions and recommendations of their healthcare professional. Thus, use of the OPTIMA questionnaire responds to the 
concern that personalised patient therapeutic objectives should be established jointly by healthcare professionals and patients, improving patient adherence to these objectives. The OPTIMA questionnaire consists of five modules, each dealing with one key area in T2DM management: PA, diet, treatment, knowledge of the disease and self-monitoring of blood glucose. The use of this questionnaire by GPs and diabetologists in their routine clinical practice and the establishment of Specific, Measurable, Acceptable, Realistic and Timely (SMART) micro-objectives following its use were recently assessed in the OPTIMA study [6]. Results of this observational study showed that most consultations (92.7\%) led to the setting up of a micro-objective concerning T2DM management by physicians and patients using the OPTIMA questionnaire. However, only $23 \%$ of these micro-objectives were SMART. Amongst the five modules of the OPTIMA questionnaire, the PA module (OPTIMA-PA) was the most likely to lead to SMART micro-objectives (34.3\%) being established, as patients were convinced that any improvement in PA would be useful, and the objectives seemed more readily attainable than those relating to diet.

The overall aim of the current OPADIA study was to assess the usefulness of the OPTIMA-PA questionnaire module for increasing the proportion of medical consultations ending with a SMART micro-objective for PA (SMART-PA micro-objective) being established through a shared decision making process. We also aimed to assess factors considered likely to have an impact on the setting up of SMART-PA microobjectives or to influence the extent of changes in PA, such as GP characteristics and the levels GP empathy perceived by the patient, and to identify any potential associations between the setting up of a SMART-PA micro-objective and/ or completing the OPTIMA-PA questionnaire module and changes in PA or drug compliance.

\section{METHODS}

\section{Study Design and Setting}

OPADIA was a multicentre, national, prospective, comparative, randomised study conducted in daily routine clinical practice in France from July 2017 to August 2018. This real-life study was conducted by GPs at participating centres through visits scheduled as part of the patients' usual care. The study was designed in collaboration with a scientific committee composed of four of the authors (SC, MD, AG, AP) who provided their expertise during the project.

In accordance with French law, approval for the processing of personal data was obtained from the French data protection agency (Commission Nationale de l'Informatique et des Libertés; CNIL). The study methodology was approved by the French advisory committee on information processing in healthcare research (Comité Consultatif sur le Traitement de l'Information en matière de Recherche dans le domaine de la Santé; CCTIRS). This study was performed in accordance with the Helsinki Declaration of 1964 and its later amendments. IRB approval was granted by Groupe Hospitalier Pitié Salpétrière (CPP82-16, ref ID RCB 2016-A01615-46).

\section{Participant Recruitment and Randomisation}

\section{Physicians}

GPs were recruited from a professional survey database: of the 7815 GPs listed in this database, 4500 were randomly selected and asked to participate in the study. Participating GPs were randomly allocated to either the standard group (patient follow-up according to the GP's usual clinical practice) or the OPTIMA-PA group (use of the OPTIMA-PA questionnaire and patient follow-up according to the GP's usual clinical practice). The GPs who participated in the study were informed of the methodology and objectives of the study and gave their written consent. GPs in both groups received training for the study, during which the value of SMART-PA micro-objectives in T2DM management was clearly explained. GPs in the OPTIMA-PA group also received specific information on the use of the OPTIMA-PA questionnaire. 


\section{Patients}

Eligible patients were adults presenting with T2DM, not currently treated with insulin [or glucagon-like peptide 1 (GLP-1) agonists] or treated with dietary and lifestyle measures only, with HbA1c levels ranging from 7.0 to $9.5 \%$, without any formal contraindications to PA, and being able to complete the self-questionnaires. Patients with type 1 diabetes and pregnant women presenting with gestational diabetes were not eligible. All patients gave their oral informed consent before inclusion, after having been informed about the study.

\section{OPTIMA-PA Questionnaire}

The PA questionnaire (OPTIMA-PA) used in this study was one of the five modules constituting the OPTIMA questionnaire [6]. This module consists of 14 items: 11 items each describing a specific type of $\mathrm{PA}$-for which the patients indicated on a five-point Likert scale how "easy" they would find the PA to do, how "useful" they considered this activity to be for controlling their T2DM and the frequency at which they thought they would be able to do this activityand three items related to a PA that the patient considered as realistically feasible. For these last three items, the patients were asked to indicate the activity name, the time during which they could do this activity and the frequency at which they could do it. Responses for these last three items were used by the GPs during the inclusion visit as a basis for discussions with their patient about PA objectives.

\section{Study Plan}

A summary of the study plan is shown in Fig. 1. The study included two visits corresponding to two usual follow-up consultations of T2DM patients: one inclusion visit and one follow-up visit $3 \pm 1$ months later. Before randomisation, all GPs completed a general practice questionnaire to provide details of their demographic and professional characteristics and evaluated their own level of PA using the Short Form of the International Physical Activity Questionnaire (IPAQ-SF, [7]) and their ability to initiate a patient-centred relationship using the Self-Efficacy in Patient-Centredness Questionnaire (SEPCQ-27, [8]). At the inclusion visit, the GPs collected patient socio-demographic and clinical data related to diabetes and its treatment, and the patients completed several self-evaluation questionnaires: part of the questionnaire used in the French national survey of people treated for diabetes (Échantillon National Témoin REprésentatif des personnes Diabétiques, ENTRED [9]) to evaluate the level of patient-physician shared decision making and the degree of physician's empathy from the point of view of the patient, the IPAQ-SF questionnaire to evaluate patient levels of PA and the GIRERD questionnaire [10] adapted for diabetes [9] to evaluate patient-reported levels compliance to treatment. Patients of GPs allocated to the OPTIMA-PA group also completed the OPTIMA-PA questionnaire, which was used by the GPs during the inclusion visit to communicate about diabetes with their patient. At the end of the inclusion visit, the GPs recorded the PA micro-objective(s) set up with their patient. During the follow-up visit, the GPs completed a follow-up questionnaire, and the patient completed the IPAQ-SF and GIRERD questionnaires. At the end of the study, two members of the scientific committee, blinded to the GPs' study group, assessed whether PA micro-objectives set up during the inclusion visits were SMART or not by using a standardised evaluation grid, established by author consensus. In case of rating discrepancies, the micro-objectives were reviewed and validated by a third member of the scientific committee.

\section{Main Outcome Measure}

The main outcome measure was the impact of the OPTIMA questionnaire on the setting up of SMART-PA micro-objectives, evaluated by a between-group comparison assessing the proportion of inclusion visits ending with at least one SMART-PA micro-objective being set up. 


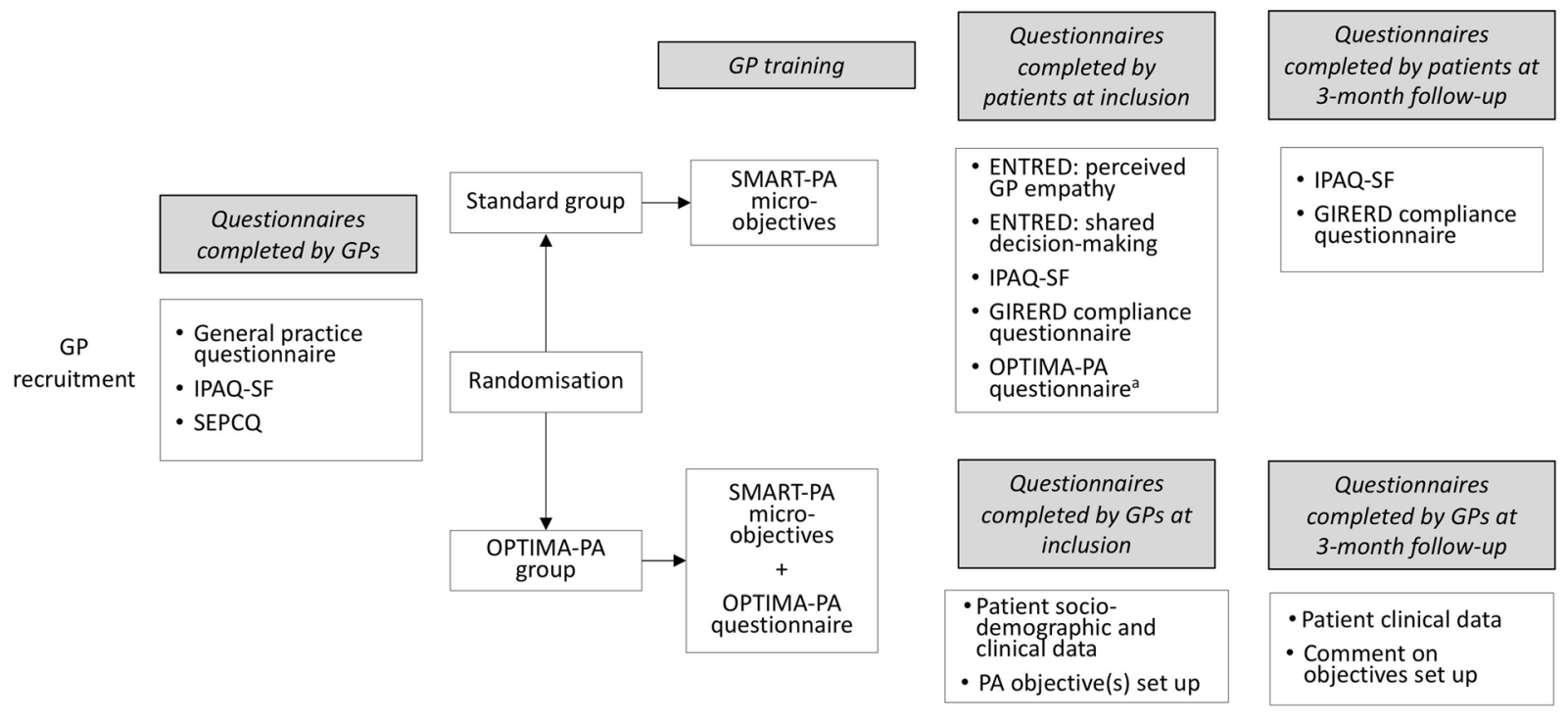

Fig. 1 Plan of the OPADIA study and evaluation timeline. ${ }^{\mathrm{a}}$ Questionnaire completed only by the patients included by GPs allocated to the OPTIMA-PA group. GP general practitioner, IPAQ-SF International Physical Activity Questionnaire Short Form, SEPCQ Self-Efficacy in Patient-Centeredness Questionnaire, SMART-PA Specific, Measurable, Acceptable, Realistic and Timely-

\section{Secondary Outcome Measures}

The following secondary outcome measures were assessed: (1) the impact of the OPTIMA-PA questionnaire and/or of setting up SMART-PA micro-objectives on changes in patient PA and compliance to treatment between the inclusion and 3-month follow-up visit, evaluated by between-group comparisons of the changes in IPAQ-SF and GIRERD scores; (2) the impact of the OPTIMA-PA questionnaire on levels of shared decision making and the degree of physician empathy, as perceived by patient, evaluated by between-group comparisons of ENTRED responses provided at inclusion; (3) the impact of patient PA levels at baseline on the setting up of at least one SMART-PA microobjective, evaluated by between-group comparisons of baseline IPAQ-SF scores; (4) the correlations between patient-perceived levels of physician empathy and physician self-reported aptitude for patient-centredness (evaluated by comparison of the ENTRED responses and SEPCQ scores); (5) the association between
Physical Activity, OPTIMA-PA group, GPs proposing their patients to complete the physical activity module of the OPTIMA questionnaire; ENTRED, Questionnaire used in the Échantillon National Témoin REprésentatif des personnes Diabétiques study, GIRERD Compliance questionnaire adapted from hypertension to diabetes

physician-reported levels of patient-centredness and/or physician PA levels and changes in patient PA levels between inclusion and the 3-month follow-up visit (evaluated by assessing correlations between SEPCQ and physician IPAQ-SF scores, and changes in patient IPAQ-SF scores of $\geq 25 \%$ ).

\section{Evaluation Questionnaires}

\section{IPAQ-SF}

The IPAQ-SF [7] is a validated self-questionnaire consisting of seven open-ended questions about an individual's PA during daily life over the past 7 days. PA is classified into three categories: low intensity activity (walking of any kind), moderate intensity activity (breathing somewhat harder than normal) and vigorous activity (hard physical effort which makes breathing much harder than normal). Total weekly PA at inclusion was estimated by weighting the reported minutes per week within each PA category by a metabolic equivalent of task (MET) energy expenditure estimate assigned to each activity 
(MET intensity) and by their frequency per week. A global PA score was calculated by summing the MET minutes per week (MET.min/ week) for each intensity category. Three categories of patients were then defined depending on their global physical score:

- inactive: $<600$ MET.min/week $(<150 \mathrm{~min}$ of moderate intensity PA);

- moderately active: $>600$ MET.min/week but $<3000$ MET.min/week (between 150 and 750 min of moderate intensity PA);

- highly active: $>3000$ MET.min/week (> 750 min of moderate intensity PA).

PA corresponding to an IPAQ score of $>600$ MET.min/week is recommended for T2DM patients.

\section{SEPCQ-27}

The SEPCQ-27 is the 27-item version of the preliminary 88-item questionnaire (SEPCQ-88) [8]. Three factors were identified as valid subscales covering core aspects of a physician's selfefficacy in patient-centredness: (1) exploring the patient's perspective, (2) sharing information and power and (3) dealing with communicative challenges. The scoring format is a fivepoint Likert scale with " 0 " (to a very low degree) and " 4 " (to a very high degree) as endpoints. A global score was calculated by summing the scores obtained for each item: the higher the score, the greater the degree of physician selfefficacy in patient-centredness. For analysis of the outcomes, these total scores were grouped into four classes: $<75,75-84,85-94$ and $\geq 95$.

\section{ENTRED}

Two simple-choice questions derived from the original ENTRED self-questionnaire [9] were used in our study: (1) In your relationship with your doctor, would you say generally that: (a) the physician decides and you follow his/her prescriptions; (b) the physician and you both decide together; (c) the physician gives you his/ her advice and you decide by yourself? (2) Would you say that you can easily discuss your concerns with your doctor? (a) absolutely; (b) mostly yes; (c) not always; (d) not at all. For this second question, answers were coded 4-1 to indicate the degree of physician empathy perceived by the patient. For analysis of the outcomes, the answers "absolutely" and "mostly yes" were grouped as characterising a high degree of physician empathy, as perceived by patient.

\section{GIRERD}

The GIRERD questionnaire $[9,10]$ is a validated self-questionnaire composed of six questions with yes/no answers: (1) Do you sometimes forget to take your medicine? (2) Have you ever run out of your medicine? (3) Do you sometimes take your medicine late? (4) Do you sometimes decide not to take your medicine because some days you feel that your treatment does more harm than good? (5) Do you think that you have too many pills to take? (6) When you feel better, do you sometimes stop taking your medicine? Scores of 0 and 1 are respectively assigned to each "no" and "yes" answer. An overall score of 0 is considered as indicating good compliance, an overall score of 1 or 2 as indicating minor noncompliance and an overall score $\geq 3$ as indicating noncompliance/poor compliance. This questionnaire, initially developed in the field of hypertension, was adapted to diabetes by Tiv et al. [9].

\section{Determination of the Sample Size}

The sample size for the study was based on the results of the OPTIMA study [6], indicating that $40 \%$ of the patients in the OPTIMA-PA group and $20 \%$ of patients in the standard group would set up a SMART-PA micro-objective. Using these estimations, a sample size of 218 patients (109 patients per group) would have been needed to conduct comparisons of proportions between the independent samples using the chi-squared test with an alpha-risk of $5 \%$ and a beta-power of 1 . Taking into account a proportion of $10 \%$ of incomplete or nonassessable files, the target number of subjects was 240 enrolled patients (120 patients/group). The required number of fully participating physicians was 60, each of them having to include approximately four patients. 


\section{Statistical Analyses}

Qualitative and ordinal variables were described using numbers and percentages of responses, quantitative variables using numbers, mean, standard deviation (SD), median, minimum and maximum. Comparisons of paired (inclusion/follow-up) qualitative variables were carried out using the McNemar chi-squared test or the Bowker test for tables of more than $2 \times 2$ modalities. Comparisons of paired quantitative variables were performed using the paired Student $t$ test or the Wilcoxon test in case of a nonnormal distribution of data. Comparisons of unpaired qualitative variables were carried out using the Pearson chi-squared test or Fisher exact test in case of small samples (i.e., theoretical frequency $<5$ ). When required, the Mantel-Haenszel chi-squared test was used to evaluate linear associations between ordinalscaled variables. When two groups were involved, means of quantitative variables were compared using the Student $t$ test with the Satterthwaite approximation method in case of unequal variances. When more than two groups were involved, quantitative variables were compared using an analysis of variance: normality and equality of variances in each group (homoscedasticity) were verified using the Shapiro-Wilk test and Levène test, respectively. Assuming that missing data were not informative, each analysis was based on the data provided, with no imputation or removal or outlying data points. The number of patients for whom data were analysed is provided for each analysis.

SAS $^{\circledR}$ software, version 9.4 (SAS Institute Inc., NC, USA), was used for all statistical analyses. For all tests, the level of significance was 5\%.

\section{RESULTS}

\section{Physician Disposition and Characteristics}

Of 4500 physicians who were contacted, 132 agreed to participate and 80 filled in the general practice questionnaire, confirmed their participation and accepted being randomly allocated to one of the management groups (Fig. 2). The
80 GPs who validated their participation practiced in all French metropolitan regions except Corsica, mainly in Ile-de-France (21.3\%), Grand-Est (16.3\%), Hauts-de-France (16.3\%) and Nouvelle-Aquitaine $(12.5 \%)$. They practiced mainly in towns $(92.5 \%)$, in medical or medical group offices $(43.8 \%$ and $56.3 \%$, respectively). None of the GPs practiced in a clinic or health centre, or for a mutual insurance company. GPs were mainly male (90\%), non-smokers $(68.8 \%)$, with a mean \pm SD age of $56.6 \pm 9.7$ years and a mean \pm SD body mass index (BMI) of $24.6 \pm 2.8 \mathrm{~kg} / \mathrm{m}^{2}$. GPs were randomly allocated to the standard group $(n=41)$ or to the OPTIMA-PA group $(n=39)$. Of these 80 GPs, 72 (35 in the standard group and 37 in the OPTIMA-PA group) were trained for the study. Overall, 54 GPs (24 in the standard group and 30 in the OPTIMA-PA group) included at least one patient (range 1-9). The sociodemographic and clinical characteristics of these fully participating GPs were similar to those of GPs who did not include any patients. GPs saw a mean of 46 T2DM patients monthly, including an average of three new T2DM patients. The professional, clinical (total level of their own PA) and behavioural (aptitude for patient-centredness, as measured by the SEPCQ) characteristics of GPs were also similar in both management groups (see Table S1; supplementary material).

\section{Patient Disposition and Characteristics}

Patient flow through the study is also presented in Fig. 2. Of the 441 eligible patients seen for their usual T2DM management visit between July 2017 and August 2018, 256 were included (122 in the standard group and 134 in the OPTIMA-PA group), of whom 251 were followed up at 3 months [118 (96.7\%) in the standard group and $133(99.3 \%)$ in the OPTIMA-PA group]. Patient socio-demographic and clinical characteristics at inclusion are summarised in Table 1. Patients were mainly male (65.2\%), aged $63.7 \pm 11.1$ years and had a BMI of $30.1 \pm 6.4 \mathrm{~kg} / \mathrm{m}^{2}$. The mean time elapsed since diabetes diagnosis was $10.5 \pm 6.7$ years and their mean $\mathrm{HbA1c}$ level was $7.8 \pm 0.6 \%$. In 


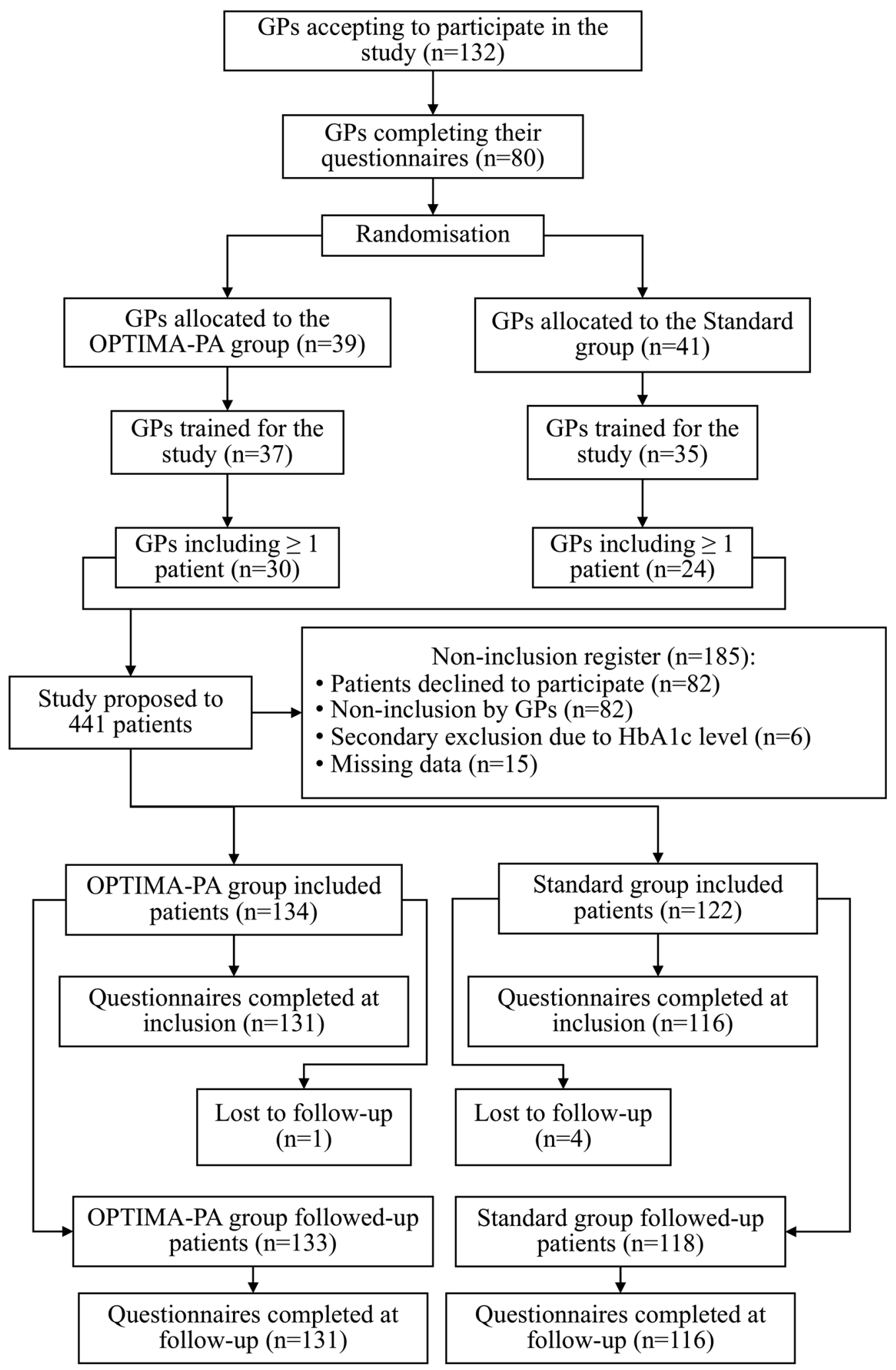

Fig. 2 Flow chart of the study 
Table 1 Patient socio-demographic and clinical characteristics at inclusion

\begin{tabular}{|c|c|c|c|c|}
\hline Parameter & $\begin{array}{l}\text { Standard } \\
(N=122)\end{array}$ & $\begin{array}{l}\text { OPTIMA-PA } \\
(N=134)\end{array}$ & $\begin{array}{l}\text { Analysed population } \\
(N=256)\end{array}$ & $p$ \\
\hline Age, years $[$ mean $( \pm S D)]$ & $63.2( \pm 10.6)$ & $64.1( \pm 11.6)$ & $63.7( \pm 11.1)$ & $0.54^{\mathrm{b}}$ \\
\hline Male, $n(\%)$ & $82(67.2)$ & $85(63.4)$ & $167(65.2)$ & $0.53^{\mathrm{c}}$ \\
\hline Education level, $n$ (\%) & & & & $0.73^{\mathrm{c}}$ \\
\hline$\leq \mathrm{CAP}, \mathrm{BEP}$ & $72(59.0)$ & $85(63.4)$ & $157(61.3)$ & \\
\hline $\begin{array}{l}\text { BAC, BAC }+1 \text { year, general or technical } \\
\text { high school }\end{array}$ & $28(23.0)$ & $24(17.9)$ & $52(20.3)$ & \\
\hline$\geq \mathrm{BAC}+2$ years & $22(18.0)$ & $25(18.7)$ & $47(18.4)$ & \\
\hline \multicolumn{5}{|l|}{ Professional status, $n(\%)$} \\
\hline Employed & $44(36.1)$ & $39(29.1)$ & $83(32.4)$ & $0.19^{\mathrm{c}}$ \\
\hline Off work (illness or accident) & $2(1.6)$ & $3(2.2)$ & $5(2)$ & \\
\hline Disabled & $6(4.9)$ & $2(1.5)$ & $8(3.1)$ & \\
\hline Unemployed & $7(5.7)$ & $14(10.4)$ & $21(8.2)$ & \\
\hline Retired (or semi-retired) & $63(51.6)$ & $76(56.7)$ & $139(54.3)$ & \\
\hline BMI, $\mathrm{kg} / \mathrm{m}^{2}[$ mean $( \pm S D)]$ & $29.9( \pm 5.6)$ & $30.4( \pm 7.1)$ & $30.1( \pm 6.4)$ & $0.53^{\mathrm{b}}$ \\
\hline Physical activity & $n=86$ & $n=89$ & $n=175$ & $0.03^{\mathrm{b}}$ \\
\hline Total PA, MET.min/week: mean \pm SD & $1098.8 \pm 1563$ & $2139 \pm 4292.8$ & $1627.8 \pm 3284$ & \\
\hline PA by tertile, $n(\%):<300$ MET.min/week & $28(32.6)$ & $34(38.2)$ & $62(35.4)$ & \\
\hline 300-1000 MET.min/week & $31(36.0)$ & $16(18.0)$ & $47(26.9)$ & $0.03^{\mathrm{c}}$ \\
\hline > 1000 MET.min/week & $27(31.4)$ & $39(43.8)$ & $66(37.7)$ & \\
\hline PA by class ${ }^{\mathrm{a}}, n(\%)$ : inactive & $43(50)$ & $40(44.9)$ & $83(47.7)$ & $0.56^{\mathrm{c}}$ \\
\hline Moderately active & $34(39.5)$ & $35(39.3)$ & $69(39.4)$ & \\
\hline Highly active & $9(10.5)$ & $14(15.7)$ & $23(13.1)$ & \\
\hline \multicolumn{5}{|l|}{ Smoking status, $n$ (\%) } \\
\hline Nonsmoker & $90(73.8)$ & $94(70.1)$ & $184(71.9)$ & $0.43^{\mathrm{c}}$ \\
\hline Former smoker & $24(19.7)$ & $25(18.7)$ & $49(19.1)$ & \\
\hline Smoker & $8(6.6)$ & $15(11.2)$ & $23(9)$ & \\
\hline HbAlc $<1$ year, & $n=116$ & $n=122$ & $n=238$ & $0.94^{\mathrm{b}}$ \\
\hline Mean $\pm S D$ & $7.8 \pm 0.6$ & $7.8 \pm 0.6$ & $7.8 \pm 0.6$ & \\
\hline LDL cholesterol $<1$ year, g/l & $n=104$ & $n=104$ & $n=208$ & \\
\hline Mean $( \pm S D)$ & $1.2( \pm 0.5)$ & $1.1( \pm 0.4)$ & $1.1( \pm 0.4)$ & $0.39^{\mathrm{b}}$ \\
\hline Systolic blood pressure, $\mathrm{mmHg}$ & $n=121$ & $n=134$ & $n=255$ & \\
\hline Mean $( \pm S D)$ & $134( \pm 11.1)$ & $130.8( \pm 9.6)$ & $132.3( \pm 10.4)$ & $0.01^{\mathrm{b}}$ \\
\hline
\end{tabular}


Table 1 continued

\begin{tabular}{|c|c|c|c|c|}
\hline Parameter & $\begin{array}{l}\text { Standard } \\
(N=122)\end{array}$ & $\begin{array}{l}\text { OPTIMA-PA } \\
(N=134)\end{array}$ & $\begin{array}{l}\text { Analysed population } \\
(N=256)\end{array}$ & $p$ \\
\hline Diastolic blood pressure, $\mathrm{mmHg}$ & $n=121$ & $n=134$ & $n=255$ & \\
\hline Mean $( \pm S D)$ & $78.5( \pm 8.5)$ & $76.8( \pm 7.5)$ & $77.6( \pm 8)$ & $0.10^{\mathrm{b}}$ \\
\hline Time since diabetes diagnosis, years & $n=121$ & $n=132$ & $n=253$ & \\
\hline Mean $( \pm S D)$ & $10.7( \pm 6.7)$ & $10.2( \pm 6.7)$ & $10.5( \pm 6.7)$ & $0.58^{\mathrm{b}}$ \\
\hline $\begin{array}{l}\text { Current treatment with oral antidiabetic } \\
\text { drugs, } n(\%)\end{array}$ & $121(99.2)$ & $131(97.8)$ & $252(98.4)$ & $0.36^{\mathrm{c}}$ \\
\hline Monotherapy & $38(31.4)$ & $42(32.1)$ & $80(31.7)$ & \\
\hline Bitherapy & $56(46.3)$ & $63(48.1)$ & $119(47.2)$ & \\
\hline Tritherapy or more & $27(22.3)$ & $26(19.8)$ & $53(21.0)$ & \\
\hline $\begin{array}{l}\text { Current treatment with a GLP-1 analogue, } \\
n(\%)\end{array}$ & $21(17.2)$ & $13(9.7)$ & $34(13.3)$ & $0.08^{\mathrm{c}}$ \\
\hline $\begin{array}{l}\text { Patient's estimated level of knowledge on } \\
\text { diabetes, } n(\%)\end{array}$ & & & & $0.69^{c}$ \\
\hline Very good & $22(18)$ & $24(17.9)$ & $46(18.0)$ & \\
\hline Good & $60(49.2)$ & $57(42.5)$ & $117(45.7)$ & \\
\hline Moderate & $34(27.9)$ & $46(34.3)$ & $80(31.3)$ & \\
\hline Poor & $6(4.9)$ & $7(5.2)$ & $13(5.1)$ & \\
\hline $\begin{array}{l}\text { Therapeutic education related to diabetes, } \\
n(\%)\end{array}$ & $26(21.3)$ & $49(36.6)$ & $75(29.3)$ & $<0.01^{\mathrm{c}}$ \\
\hline $\begin{array}{l}\text { Complications associated with diabetes, } \\
n(\%)\end{array}$ & $27(22.1)$ & $34(25.4)$ & $61(23.8)$ & $0.54^{\mathrm{c}}$ \\
\hline \multirow[t]{2}{*}{ Other comorbidities, $n(\%)$} & $n=122$ & $n=133$ & $n=255$ & \\
\hline & $99(81.1)$ & $105(78.9)$ & $204(80)$ & $0.66^{\mathrm{c}}$ \\
\hline
\end{tabular}

$B A C$ baccalauréat (bachelor degree), $B M I$ body mass index, $B E P$ brevet d'études professionnelles (vocational high school), CAP certificat d'aptitude professionnelle (Youth Training [NVQ Level 1, 2]), GLP-1 glucagon-like peptide 1, HbAIc glycated haemoglobin, $L D L$ low density lipoprotein, $M E T$ metabolic equivalent of task, $S D$ standard deviation

a PA classes: inactive, $<600 \mathrm{MET} . \mathrm{min} /$ week $(<150 \mathrm{~min}$ of moderate intensity PA); moderately active: $600<$ total $\mathrm{PA}<3000 \mathrm{MET}$.min/week (between 150 and $750 \mathrm{~min}$ of moderate intensity PA); highly active $>3000 \mathrm{MET}$.min/week $\left(>750\right.$ min of moderate intensity PA). $p$ values were obtained using the Student $t$ test $^{\mathrm{b}}$ or a chi-squared test ${ }^{\mathrm{c}}$

total, $23.8 \%$ of patients had complications associated with their T2DM and $80.0 \%$ had comorbidities other than those linked to diabetes. Overall, T2DM was managed with lifestyle changes in only $1.6 \%$ of patients and with oral antidiabetic agents (OADs) in $98.4 \%$ of patients. There were no significant differences between the standard group and the OPTIMAPA group for any of these characteristics, except for systolic blood pressure-which was higher 
in the standard group $(p=0.01)$-and two characteristics of interest for the study. First, the percentage of patients who received therapeutic education related to diabetes was higher in the OPTIMA-PA group than in the standard group (36.6\% versus $21.3 \%, p<0.01)$. Second, patients in the OPTIMA-PA group were more active, with a higher mean total PA score at inclusion, than those in the standard group $(p=0.03)$.

\section{Proportion of SMART-PA Micro-objectives Set Up During the Inclusion Visit}

In the total population, $95.3 \%$ of inclusion visits $(n=244 / 256)$ ended with an objective (SMART or not SMART) being established, with $69.9 \%$ of inclusion visits $(n=179 / 256)$ ending with the setting up of at least one SMART-PA micro-objective. The rate of inclusion visits ending with the setting up of at least one SMART-PA micro-objective was higher in the standard group than in the OPTIMA-PA group: $81.1 \%(n=99 / 122)$ versus $59.7 \%(n=80 / 134)$, respectively $(p<0.001)$. Amongst patients for whom at least one objective (SMART or not

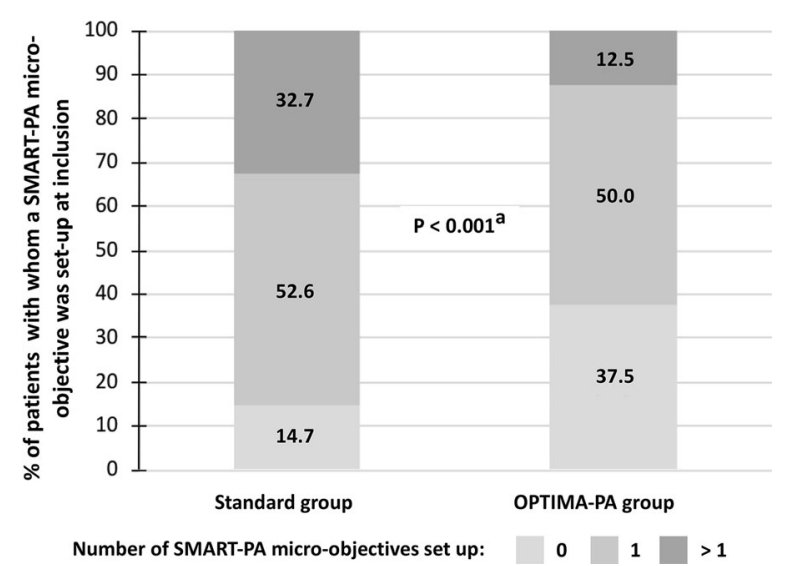

Fig. 3 Percentages of inclusion visits ending with the setting up of 0,1 or $>1$ SMART-PA micro-objectives. Only patients in each group with whom at least one objective was established at the inclusion visit were included in this analysis $(n=116$ for the standard group and $n=128$ for the OPTIMA-PA group. ${ }^{\text {a }} p$ value for between-group comparisons obtained using the MantelHaenszel chi-squared test for ordinal-scaled variables. $P A$ physical activity
SMART) was established at the inclusion visit ( $n=116$ for the standard group and $n=128$ for the OPTIMA-PA group; Fig. 3), the rate of visits ending with just one SMART-PA micro-objective being set up was similar in both groups $(52.6 \%$ versus $50.0 \%)$, the rate of visits ending with more than one SMART-PA micro-objective being set up was higher in the standard group than in the OPTIMA-PA group $(32.7 \%$ versus $12.5 \%)$ and the rate of visits ending with no SMART-PA micro-objective being set up was significantly higher in the OPTIMA-PA group than in the standard group $(37.5 \%$ versus $14.7 \%, p<0.001)$.

\section{Analysis of Baseline Patient PA Levels and Their Association with SMART-PA Micro-objectives}

In the OPTIMA group, the setting up of at least one SMART-PA micro-objective was not significantly associated with the class of PA of the patients (inactive, moderately active, or highly active) at inclusion. However, a trend emerged for the standard group $(p=0.06)$ for which the lower the PA level of the patients at inclusion, the higher the number of patients with at least one SMART-PA micro-objective set up (Fig. 4).

This trend was supported by an analysis of intense PA: total duration of vigorous PA during the week before inclusion and total score for vigorous PA were significantly lower in patients for whom at least one SMART-PA micro-objective was set up at inclusion than in those in which no SMART-PA micro-objective was established $\quad(46.5 \pm 161.5$ versus $177.5 \pm$ $466.5 \mathrm{~min} /$ week, $p=0.04$, and $372.1 \pm 1292.2$ versus $1419.7 \pm 3732$ MET.min/week, $p=0.04$, respectively) in both groups. However, according to the multivariate logistic regression analysis, the only independent predictive factor for the setting up of at least one SMART-PA microobjective was the GPs' randomisation group.

\section{Change in Patient PA levels Between Inclusion and Follow-Up}

For patients who completed the IPAQ-SF questionnaires at inclusion and at follow-up, a clear 


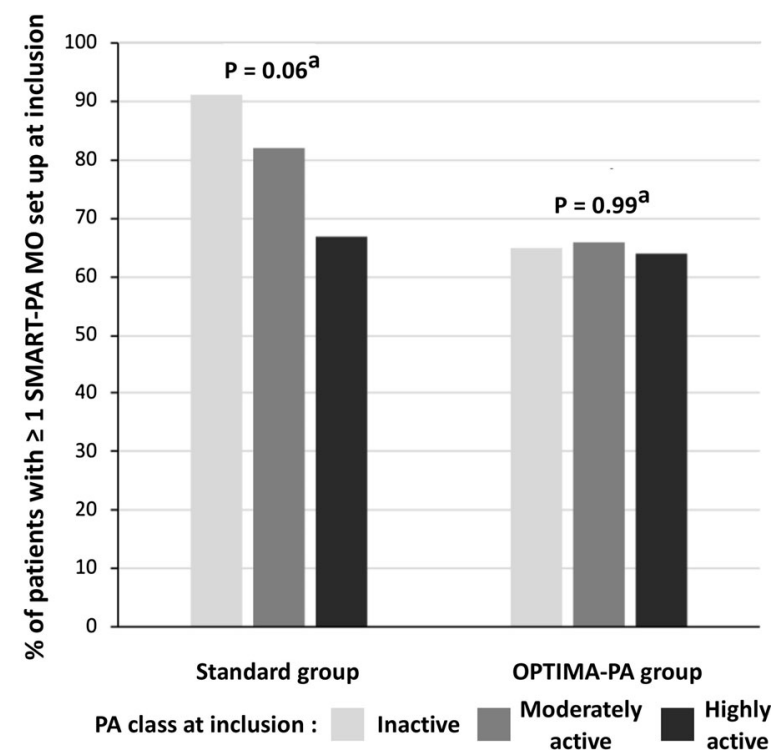

Fig. 4 Percentage of patients with at least one SMARTPA micro-objective according to the class of patient physical activity level at inclusion. ${ }^{a} p$ value obtained using the Mantel-Haenszel chi-squared test for ordinal-scaled variables. Inactive: $<600 \mathrm{MET}$.min/week $(<150 \mathrm{~min}$ of moderate intensity physical activity); moderately active: between 600 and $3000 \mathrm{MET}$.min/week (between 150 and $750 \mathrm{~min}$ of moderate intensity physical activity); highly active: $>3000$ MET.min/week (>750 min of moderate intensity physical activity). $P A$ physical activity, $M O$ micro-objective

increase in MET.min/week total PA over the 3-month study period was observed in patients for whom at least one SMART-PA micro-objective was set up at the inclusion visit as well as for those in the standard group $(p<0.001$ for both; Fig. 5). When comparing the PA change between groups, the increase in PA tended to be larger in the standard group than in the OPTIMA-PA group $(p=0.07)$ and in patients with whom at least one SMART-PA micro-objective was set up at inclusion $(p=0.09)$ (Table 2; Fig. 5); however, this increase only reached significance in the standard subgroup $(p<0.01$; Table 2$)$. Moreover, the change in PA between inclusion and follow-up was especially large when the patient level of PA at inclusion was low or close to the lower limit of recommended levels: using a median split of the distribution of patient PA at inclusion, the proportion of patients with an increase in PA of at least $25 \%$ was significantly higher when PA at inclusion was $<800 \mathrm{MET} \mathrm{min} /$ week than when $\mathrm{PA}$ at inclusion was $\geq 800 \mathrm{MET} \mathrm{min} /$ week (87.3\% versus $38.2 \%, p<0.0001$ ).

\section{Patient Compliance to Treatment}

Overall scores for the GIRERD questionnaire revealed minor noncompliance and poor compliance for respectively $42.4 \%(n=98 / 231)$ and $21.6 \%(n=50 / 231)$ of patients at inclusion, and $40.2 \%(n=84 / 209)$ and $12.9 \%(n=27 / 209)$ of patients at follow-up. There were no betweengroup differences in any change in compliance $(p=0.69)$. Similarly, the change in compliance between inclusion and follow-up was not significantly associated with the setting up of a SMART-PA micro-objective $(p=0.33)$.

\section{Shared Therapeutic Decision Making, Patient-Perceived Physician Empathy and GP-Reported Patient-Centeredness}

Patient perceptions of the degree of patientphysician shared decision making are presented in Fig. 6. Perceptions were significantly different between groups $(p<0.0001)$, with a higher proportion of patients in the standard group than in the OPTIMA group feeling that decisions were not shared: a lack of shared decision making was reported by $59.6 \%$ of patients $(n=68 / 114)$ from the standard group versus $32 \%$ of patients $(n=40 / 125)$ from the OPTIMAPA group, $p<0.0001)$. The perception of shared decision making was not associated with the setting up of at least one SMART-PA micro-objective at inclusion $(p=0.72)$.

Most patients perceived a high degree of empathy from their GP in both the standard and OPTIMA-PA groups: $74.6 \% \quad(n=85 / 114)$ and $71.7 \% \quad(n=91 / 127), \quad$ respectively. The degree of GP empathy perceived by the patient was linearly associated with GP-reported levels of patient-centredness (total SEPCQ score in four classes, $p<0.0001$; Fig. 7). Moreover, the total GP SEPCQ score in the four classes was significantly associated with a gradual increase in total patient PA levels of at least 25\% between inclusion and follow-up, $p=0.003$ 


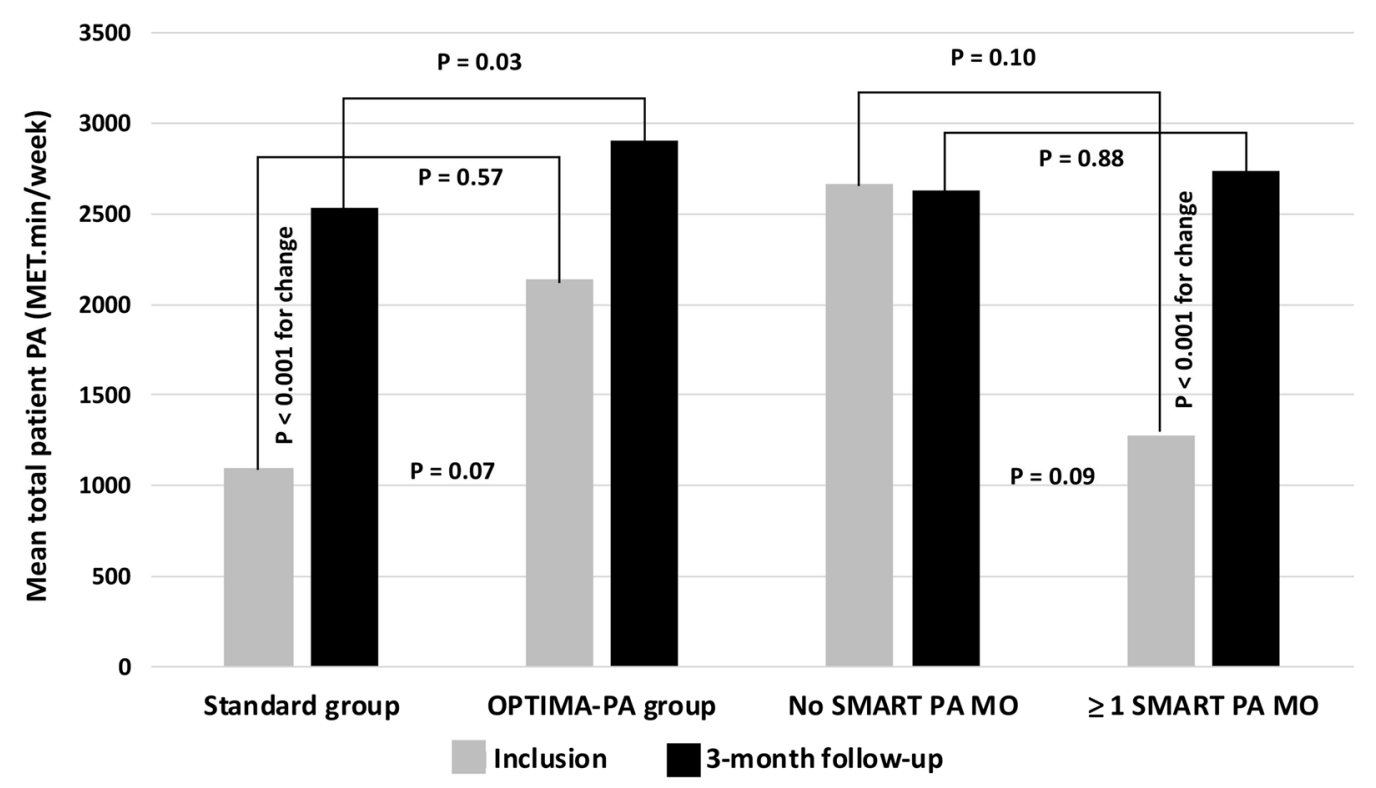

Fig. 5 Total patient physical activity at inclusion and follow-up according to the study group and as to whether a conducted using Student tests for paired or unpaired groups. $P A$ physical activity, $M E T$ metabolic equivalent of SMART-PA objective was established. Analyses were task, $M O$ micro-objective

Table 2 Changes in physical activity level from inclusion to 3 months according to the study group (OPTIMA-PA or standard) and as to whether a SMART-PA micro-objective was established at inclusion

\begin{tabular}{|c|c|c|c|c|c|c|}
\hline & $\begin{array}{l}\text { Standard } \\
(N=119)\end{array}$ & $\begin{array}{l}\text { OPTIMA } \\
(N=132)\end{array}$ & $p$ & $\begin{array}{l}\text { SMART-PA } \\
\text { microobjective } \\
(N=176)\end{array}$ & $\begin{array}{l}\text { NO SMART-PA } \\
\text { microobjective } \\
(N=75)\end{array}$ & $p$ \\
\hline \multicolumn{7}{|c|}{ Change in total PA (MET.min/week) } \\
\hline Analysed, $n(\%)$ & $71(59.7)$ & $59(44.7)$ & & $101(57.4)$ & $29(38.7)$ & \\
\hline Mean $\pm S D$ & $1413.3 \pm 3024$ & $448.2 \pm 2933.2$ & $0.07^{\mathrm{a}}$ & $1218.5( \pm 2965)$ & $128.3( \pm 3065.5)$ & $0.09^{\mathrm{a}}$ \\
\hline Median (min-max) & $\begin{array}{r}546(-1680 \\
\text { to } 15,093)\end{array}$ & $\begin{array}{c}396(-14,223 \\
\text { to } 5139)\end{array}$ & & $\begin{array}{l}651(-8610 \text { to } \\
15,093)\end{array}$ & $\begin{array}{l}330(-14,223 \text { to } \\
5139)\end{array}$ & \\
\hline $\begin{array}{l}\text { Change in total PA in the } \\
\text { standard group } \\
\text { (MET.min/week) }\end{array}$ & & & & $n=61$ & $n=10$ & \\
\hline Mean $\pm S D$ & & & & $1620,3( \pm 3212)$ & $150,6( \pm 542)$ & $<0.01^{2}$ \\
\hline Median (min-max) & & & & $\begin{array}{l}702(-1680 \text { to } \\
15,093)\end{array}$ & $\begin{array}{l}66(-729 \text { to } \\
1017)\end{array}$ & \\
\hline $\begin{array}{l}\text { Change in total PA in the } \\
\text { OPTIMA group } \\
\text { (MET.min/week) }\end{array}$ & & & & $n=40$ & $n=19$ & \\
\hline Mean $\pm S D$ & & & & $605.8( \pm 2457.2)$ & $116.5( \pm 3804.1)$ & $0.61^{\mathrm{a}}$ \\
\hline
\end{tabular}


Table 2 continued

\begin{tabular}{llllll}
\hline & $\begin{array}{l}\text { Standard } \\
(\boldsymbol{N}=\mathbf{1 1 9})\end{array}$ & $\begin{array}{l}\text { OPTIMA } \\
(\boldsymbol{N}=\mathbf{1 3 2})\end{array}$ & $\boldsymbol{p}$ & $\begin{array}{l}\text { SMART-PA } \\
\text { microobjective } \\
(\boldsymbol{N}=\mathbf{1 7 6})\end{array}$ & $\begin{array}{l}\text { NO SMART-PA } \\
\text { microobjective } \\
(\boldsymbol{N}=75)\end{array}$
\end{tabular}

$P A$ physical activity, MET metabolic equivalent of task. $p$ values were obtained using the Student $t$ test ${ }^{\mathrm{a}}$ or a Chi-squared test $^{\mathrm{b}}$

(Fig. 8). The relationship between the GP SEPCQ score and an increase in total patient PA levels of at least $25 \%$ persisted even after adjusting for the study group of the patients, $p=0.003$ (standard or OPTIMA-PA group).

\section{Impact of GP Levels of PA on the Setting Up of SMART-PA Micro-objectives and the Change in Patient Levels of PA}

The percentage of patients with whom at least one SMART-PA micro-objective was set up and the percentage of patients who increased their PA level by $25 \%$ between inclusion and followup did not differ significantly according to the PA level of their GP $(p=0.36$ and $p=0.24$, respectively; Table S2, supplementary information).

\section{DISCUSSION}

This multicentre, prospective, comparative randomised study carried out in a real-life setting in French GP practices failed to demonstrate that use of the OPTIMA-PA

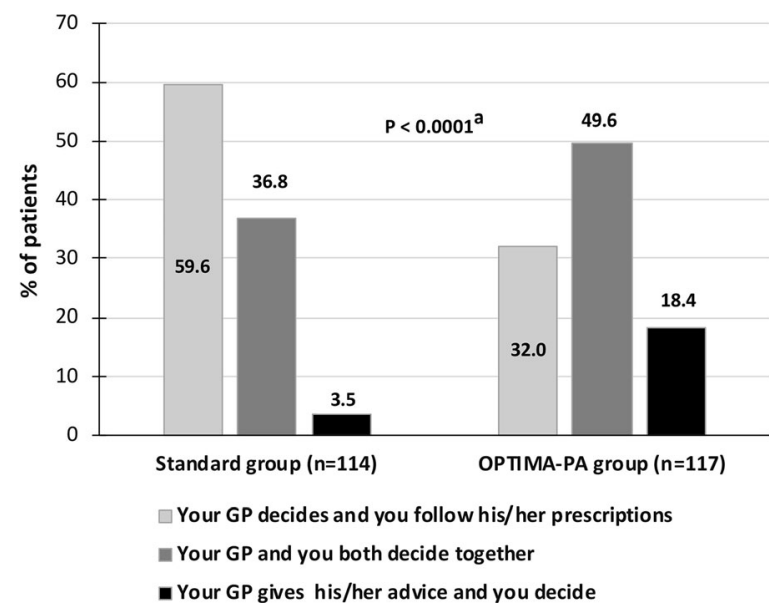

Fig. 6 Patient perception of the level of shared decision making (ENTRED questionnaire) according to the study group. ${ }^{a} p<0.0001$ for the difference between groups analysed using the Mantel-Haenszel chi-squared test for ordinal-scaled variables. GP general practitioner, $P A$ physical activity 


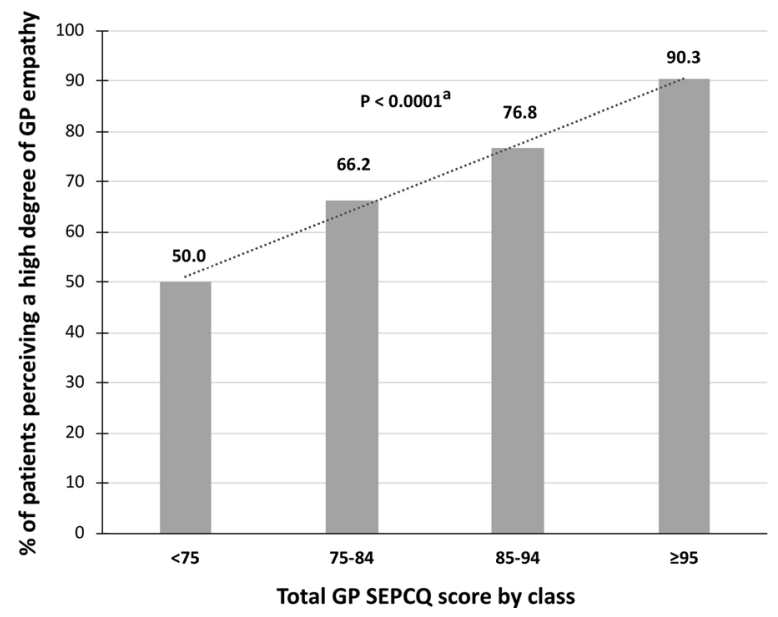

Fig. 7 GP empathy perceived by the patients (ENTRED questionnaire) according to the degree of self-efficacy in patient-centredness of their GP (total SEPCQ score). ${ }^{\mathrm{a}} p<0.0001$ for the association between patient-perceived GP empathy and total SEPCQ score when analysed using the Mantel-Haenszel chi-squared test for ordinal-scaled variables. GP general practitioner, ENTRED questionnaire used in the Echantillon National Témoin REprésentatif des personnes Diabétiques study, SEPCQ Self-Efficacy in Patient-Centredness Questionnaire

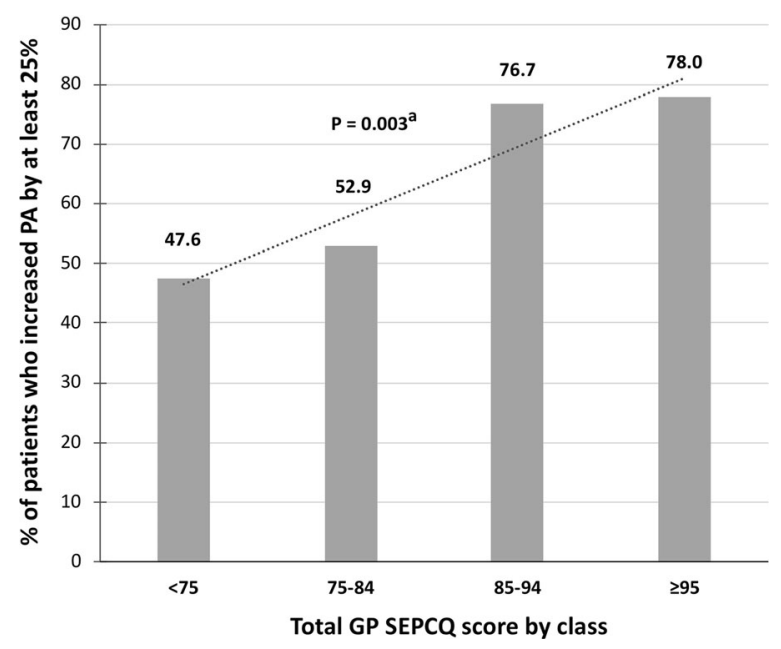

Fig. 8 Percentage of patients who increased their physical activity by at least $25 \%$ at 3 -month follow-up according to the total GP SEPCQ score by class. ${ }^{\mathrm{a}} p=0.003$ for the association between total GP SEPCQ score and an increase of at least $25 \%$ in patient physical activity levels when analysed using the Mantel-Haenszel chi-squared test for ordinal-scaled variables. $P A$ physical activity, SEPCQ Self-Efficacy in Patient-Centredness Questionnaire questionnaire improved the likelihood of SMART-PA micro-objectives being set up for diabetic patients. Unexpectedly, we found that SMART-PA micro-objectives were more often established in patients managed by standard practice. However, the OPTIMA-PA questionnaire proved to be a valuable aid for bettering GP communication with their patients, and for improving patient relationships with their GP, leading to increased perceptions of shared decision making. GP self-efficacy in engaging in a patient-centred relationship was associated with greater levels of patient-perceived physician empathy and, interestingly, with an increase in patient PA at follow-up, independently of the setting up of SMART-PA microobjectives.

The patients included in our study were representative of the French adult population with T2DM and compared well with those in the ENTRED study: in terms of mean age (64 years versus 65 years), male prevalence (65\% versus $54 \%$ ) and median time since diagnosis of T2DM (10 years and 9 years) $[9,11,12]$. Diabetes in the OPADIA patients was frequently associated with complications (around 25\% of the population). The majority of patients included in the OPADIA study were treated with OADs, often prescribed as a dual therapy (47\% of patients) or monotherapy (32\% of patients), with biguanide being the most commonly prescribed molecule, in line with previously published studies and recommendations on T2DM $[9,11,13]$. In terms of patient PA, levels in the OPTIMA-PA group were closer to those of patients with T2DM reported in the ENTRED study (2139 and 2079 MET.min/week, respectively) than those in the standard group (1098.8 MET.min/week) [12].

Contrary to our expectations, a greater proportion of visits in the standard group ended with SMART-PA micro-objectives being set up compared with those in the OPTIMA-PA group ( $81 \%$ versus $60 \%$, respectively). However, it should be pointed out that the overall percentage of visits ending with SMART-PA micro-objectives being set up by physicians in both groups was especially high in our study $(69.9 \%$, i.e., nearly twice as many as in the OPTIMA study) [6]. This unexpected result could be 
explained by the stimulating effect on physicians from both groups of participating in a study and by the fact that both groups received training on the value of SMART micro-objectives before the start of the study. The higher rate of SMART-PA micro-objectives in the standard group could be explained by a "competition effect" for this group specifically, for which the absence of the OPTIMA-PA tool led to greater motivation and commitment towards establishing SMART micro-objectives by the physician. This situation has been well described in previous comparative control/intervention group studies and may be similar to the socalled 'Hawthorne' effect describing the situation in which behaviours change when individuals feel observed [14]. Indeed, both groups of physicians had similar professional characteristics, received the same training for establishing SMART-PA micro-objectives and were aware that the setting up of SMART-PA microobjectives was the primary objective of the study. Conversely, physicians in the OPTIMAPA group may have focused more on the OPTIMA-PA tool itself, being more concerned with reviewing the questionnaire and engaging in discussions with their patients to develop their knowledge about diabetes and better understand their lifestyle and preferences, rather than focusing on setting up SMART-PA micro-objectives, which may have been perceived as incidental. In addition, it should be pointed out that the same proportion of visits (52.6\% and 50\%) in both groups ended with a single SMART-PA objective being established, whereas more visits in the standard group ended with the setting up of several SMART-PA micro-objectives (32.7 versus $12.5 \%$ ). However, establishing several objectives (SMART or not) at the same time for a single patient somewhat contradicts the sense of the SMART objective itself and may be counterproductive, because the attention of the patient may drift between multiple objectives and the patient may be overwhelmed or discouraged by the need to reach several goals at the same time. Physicians in the standard group tended to set up more SMART-PA micro-objectives if the PA level of their patients at inclusion was low. This correlation was not observed in the OPTIMA-PA group. Conversely, GPs in the OPTIMA-PA group may have respected the motivation and preferences of their patient, based on the OPTIMA-PA questionnaire, to make the best decision jointly with their patient on one specific agreed upon objective. This hypothesis is supported by the fact that more patients in the OPTIMA-PA group than in the standard group (68\% versus $40 \%$ ) thought that decision making was shared with their GP or even that they made decisions themselves after listening to the advice of their GP.

Another factor that may explain the difference in the SMART-PA micro-objective prescriptive behaviour of physicians in the two groups is the fact that patients in the OPTIMAPA group were globally more active at inclusion than patients in the standard group, thus setting up of a SMART-PA micro-objective might not have seemed an absolute priority for patients in the OPTIMA-PA group. However, our results showed that the GPs' randomisation group was the only independent predictive variable influencing the setting up of at least one SMART-PA micro-objective, indicating that GPs in the standard group were more likely to set up a SMART-PA micro-objective regardless of the level of patient PA at inclusion. Thus, the difference in PA between the two groups at baseline did not appear to have been a major factor in determining the number of SMART-PA micro-objectives established in each group.

When SMART-PA micro-objectives were set up, they appeared to be effective: patients with SMART-PA micro-objectives presented with an increase in PA at the 3-month follow-up visit $(p<0.001)$, and more patients with SMART-PA micro-objectives upgraded to a higher class of PA level at 3 months, although this difference did not reach statistical significance (39\% versus $17 \%, p=0.07)$.

Finally, the rate of data collection at 3 months may also have influenced our findings, with higher rates of data collection at 3 months perhaps reflecting a higher degree of mobilisation of GPs from the standard group than from the OPTIMA-PA group. Indeed, more data were available for analysis for patients with changes in their PA level in the standard group 
than in the OPTIMA-PA group $(59.6 \%$ versus $44.7 \%$ with PA analysable at 3 months).

In summary, it seems that GPs in the standard group put more emphasis on incentivising patients with low PA levels and obtained more IPAQ-SF evaluations at 3 months than GPs in the OPTIMA-PA group.

The OPTIMA-PA questionnaire was designed to favour exchanges between the physician and their patient and the results from our study show that this tool did help to improve the levels of physician-patient shared decision making (patients in the OPTIMA-PA group reported a greater sense of shared decision making, with GPs deciding alone in only $32 \%$ of cases, versus $60 \%$ of cases in the standard group). In addition, GP self-efficacy for engaging in a patient-centred relationship was itself positively correlated with patient-perceived physician empathy: the higher the SEPCQ score, the more patients reported a positive sense of physician empathy $(p<0.001)$. Similarly, the more patient-centred the relationship, the higher the proportion of patients with an increase in PA of $\geq 25 \%$, the relationship being linear $(p=0.003)$. Although the presence of a patient-centred relationship seemed to favour a positive change in PA at 3 months, it did not appear to be linked to the setting up of SMARTPA micro-objectives, but did lead to improved communication between the patient and the physician. It is questionable whether the progress in PA of the standard group is likely to be sustained over time, given the more physicianpatient directive relationship observed in this group. In contrast, in the OPTIMA-PA group, the increased sense of sharing decisions on PA objectives may likely ensure more stable outcomes over time. Longer follow-up data would be needed to answer this question.

As regards the other characteristics of the physicians, their own level of PA was not found to be predictive of a greater progression in patient PA at 3 months. These findings are in contrast with previous data from Duclos et al. [15] and Lanhers et al. [16]. In addition, none of the other characteristics of the investigators evaluated in our study appeared to influence the setting up of SMART-PA micro-objectives or the progression of patient PA.
We acknowledge several limitations of our study. There was no specific training on the OPTIMA-PA tool, which may have limited the facilitating effect of using the OPTIMA-PA questionnaire on PA progression, whereas the two groups of physicians received the same explanations on the value of SMART-PA microobjectives. Several other biases may have influenced the results. (1) The rating of SMART/nonSMART objectives focused essentially on "specific" and "measurable" criteria, which could explain the high percentage of SMART PA micro-objectives. (2) Due to the randomisation process being based on physicians, there were differences between the two groups in baseline patient characteristics, most importantly there were between-group differences in the central endpoint of the study, PA levels, and in participation in therapeutic education programs. Patients enrolled in the OPTIMA-PA group were in better physical condition and better informed on their diabetes than those of the standard group. This selection bias may explain why the setting up of SMART-PA micro-objectives in such patients may have seemed less of a priority to the physician. (3) Finally, the number of patients with missing data on PA evolution at follow-up was lower in the standard group, which may have reflected a greater degree of mobilisation of GPs in this group than in the OPTIMA-PA group.

We also acknowledge two other potential limitations. First, even though the patients with T2DM included in our study were representative of the T2DM population in France and their therapeutic management was as expected, the behaviour of physicians and patients may differ in other countries and thus the generalisability of our results to T2DM management in other countries may be limited. Second, there is currently no gold-standard criterion for measuring PA. Methods that would allow an accurate and objective measurement of PA-such as the doubly labelled water technique [15] and movement sensors such as accelerometers-are expensive and thus not usually used in largescale cohort studies [17]. Alternatively, various physical capacity measures (e.g., maximum oxygen uptake, $\mathrm{VO}_{2 \max }$ or the 6-min walking or hand grip strength test) could be used. 
However, questionnaires are frequently used to obtain PA data and the IPAQ-SF is a validated tool [7].

\section{CONCLUSIONS}

In conclusion, the OPADIA study supports the usefulness of the OPTIMA-PA questionnaire for working with T2DM patients on their objectives in a spirit of shared decision making and proved that this tool was associated with an increase in the degree of patient-perceived involvement in the making of therapeutic decisions. However, in contrast to what was expected, more SMARTPA micro-objectives were set up with patients of the standard group. Nevertheless, this study highlights the value of physician empathy and their ability to develop patient-centred relationships for improving patient PA levels. Although results of the study suggest that introducing a tool such as the OPTIMA questionnaire in the care of patients with T2DM is not sufficient, use of the questionnaire may help the patients to have a more positive perception of their relationship with their GP and contribute to their empowerment in the management of their health [18]. This questionnaire may prove to be a useful tool for improving therapeutic relationships, providing there is synergy between the level of GP empathy and their efficacy in engaging in a patient-centred relationship. Our results are in accordance with the recent European and American consensus guidelines on the management of T2DM in adults, which emphasise the usefulness of SMART micro-objectives, the central role of patient-centred decision making and support [19], and would suggest the interest of developing empathy training programmes for physicians.

\section{ACKNOWLEDGEMENTS}

We would like to acknowledge Robert (Bobby) Zachariae, professor at the Department of Psychology and Behavioural Science, Aarhus University, and the Department of Oncology, Aarhus University Hospital, for the use of his
"Self-Efficacy in Patient-Centredness Questionnaire" (SEPCQ). We would also like to thank all patients and physicians who participated in this study

Funding. Sponsorship for this study the journals rapid service fee and open access fee were funded by MSD France.

Medical Writing and Editorial Assistance. Assistance in medical writing and language editing was provided by Laurence Rous, Marielle Romet, and Emma Pilling of Santé Active Edition.

Authorship. All named authors meet the International Committee of Medical Journal Editors (ICMJE) criteria for authorship for this article, take responsibility for the integrity of the work as a whole, and have given their approval for this version to be published.

Disclosures. Sebastien Bineau and Sylvie Dejager are employees of MSD France, Bénédicte Sabin and Mathilde Pouriel are employees of Kappa Santé and Eva Freund is an employee of Clintec International. Silla Consoli, Martine Duclos, André Grimaldi, and Alfred Penfornis have nothing to disclose.

Compliance with Ethics Guidelines. In accordance with French law, approval for the processing of personal data was obtained from the French data protection agency (Commission Nationale de l'Informatique et des Libertés; CNIL). The study methodology was approved by the French advisory committee on information processing in healthcare research (Comité Consultatif sur le Traitement de l'Information en matière de Recherche dans le domaine de la Santé; CCTIRS). This study was performed in accordance with the Helsinki Declaration of 1964 and its later amendments. IRB approval was granted by Groupe Hospitalier Pitié Salpétrière (CPP82-16, ref ID RCB 2016-A01615-46).

Data Availability. All data generated or analyzed during this study are included in this published article or as supplementary information files. 
Open Access. This article is licensed under a Creative Commons Attribution-NonCommercial 4.0 International License, which permits any non-commercial use, sharing, adaptation, distribution and reproduction in any medium or format, as long as you give appropriate credit to the original author(s) and the source, provide a link to the Creative Commons licence, and indicate if changes were made. The images or other third party material in this article are included in the article's Creative Commons licence, unless indicated otherwise in a credit line to the material. If material is not included in the article's Creative Commons licence and your intended use is not permitted by statutory regulation or exceeds the permitted use, you will need to obtain permission directly from the copyright holder.To view a copy of this licence, visit http://creativecommons.org/licenses/by$\mathrm{nc} / 4.0 /$.

\section{REFERENCES}

1. Patrice Darmon P, Bauduceau B, Lyse Bordier L, Bringer J, Chabrier G, Charbonnel B, et al. Management of hyperglycemia in type 2 diabetes: position statement of the Francophone Diabetes Society. Médecine des maladies Métaboliques. 2017;11(6):577-93.

2. WHO. Global report on diabetes. Geneva: World Health Organization; 2016.

3. Duclos M, Oppert JM, Verges B, Coliche V, Gautier JF, Guezennec Y, et al. Physical activity and type 2 diabetes. Recommandations of the SFD (Francophone Diabetes Society) diabetes and physical activity working group. Diabetes Metab. 2013;39(3): 205-16.

4. Colberg SR, Sigal RJ, Yardley JE, Riddell MC, Dunstan DW, Dempsey PC, et al. Physical activity/exercise and diabetes: a position statement of the American Diabetes Association. Diabetes Care. 2016;39(11):2065-79.

5. Greenfield S, Kaplan SH, Ware JE Jr, Yano EM, Frank HJ. Patients' participation in medical care: effects on blood sugar control and quality of life in diabetes. J Gen Intern Med. 1988;3(5):448-57.

6. Grimaldi A, Penfornis A, Consoli S, Falissard B, Eymard E, Williams $\mathrm{P}$, et al. Breaking barriers to effective type 2 diabetes management: findings from the use of the OPTIMA(c) questionnaire in clinical practice. Adv Ther. 2016;33(6):1033-48.

7. Lee PH, Macfarlane DJ, Lam TH, Stewart SM. Validity of the international physical activity questionnaire short form (IPAQ-SF): a systematic review. Int J Behav Nutr Phys Act. 2011;8:115.

8. Zachariae R, O'Connor M, Lassesen B, Olesen M, Kjaer LB, Thygesen M, et al. The self-efficacy in patient-centeredness questionnaire-a new measure of medical student and physician confidence in exhibiting patient-centered behaviors. BMC Med Educ. 2015;15:150.

9. Tiv M, Viel JF, Mauny F, Eschwege E, Weill A, Fournier C, et al. Medication adherence in type 2 diabetes: the ENTRED study 2007, a French Population-Based Study. PLoS ONE. 2012;7(3):e32412.

10. Girerd X, Hanon O, Anagnostopoulos K, Ciupek C, Mourad JJ, Consoli S. Assessment of antihypertensive compliance using a self-administered questionnaire: development and use in a hypertension clinic [Article in French]. Presse Med. 2001;30(21): 1044-8.

11. Invs. Résultats épidémiologiques principaux d'Entred-métropole-Etude Entred 2007-2010. 2019. https://invs.santepubliquefrance.fr/Dossiers-thema tiques/Maladies-chroniques-et-traumatismes/Diabe te/Etudes-Entred/Etude-Entred-2007-2010/Resultatsepidemiologiques-principaux-d-Entred-metropole. Accessed 26 Mar 2020.

12. Cloix L, Caille A, Helmer C, Bourdel-Marchasson I, Fagot-Campagna A, Fournier C, et al. Physical activity at home, at leisure, during transportation and at work in French adults with type 2 diabetes: the ENTRED physical activity study. Diabetes Metab. 2015;41(1):37-44.

13. HAS. Recommandation de bonne pratique-Stratégie médicamenteuse du contrôle glycémique du diabète de type 2. 2013. https://www.has-sante.fr/ portail/upload/docs/application/pdf/2013-02/10irp 04_reco_diabete_type_2.pdf. Accessed 26 Mar 2020.

14. Oswald D, Sherrat F, Smith S. Handling the Hawthorne effect: The challenges surrounding a participant observer. Review of Social Studies. 2014;1(1).

15. Duclos M, Dejager S, Postel-Vinay N, di Nicola S, Quere S, Fiquet B. Physical activity in patients with type 2 diabetes and hypertension-insights into motivations and barriers from the MOBILE study. Vasc Health Risk Manag. 2015;11:361-71.

16. Lanhers C, Duclos M, Guttmann A, Coudeyre E, Pereira B, Ouchchane L. General practitioners' barriers to prescribe physical activity: the dark side of the cluster effects on the physical activity of their 
type 2 diabetes patients. PLoS ONE. 2015;10(10): e0140429.

17. Freedson P, Miller K. Objective monitoring of physical activity using motion sensors and heart rate. Res Q Exerc Sport. 2000;71:S21-S30.

18. Holmstrom I, Roing M. The relation between patient-centeredness and patient empowerment: a discussion on concepts. Patient Educ Couns. 2010;79(2):167-72.

19. Davies MJ, D'Alessio DA, Fradkin J, Kernan WN, Mathieu C, Mingrone G, et al. Management of hyperglycaemia in type 2 diabetes, 2018. A consensus report by the American Diabetes Association (ADA) and the European Association for the Study of Diabetes (EASD). Diabetologia. 2018;61(12):2461-98. 